\title{
Thermal response of high-aspect-ratio hydrogen cylinders undergoing fast-filling
}

\author{
V. Ramasamy ${ }^{a, *}$, E.S. Richardson ${ }^{b}$ \\ ${ }^{a}$ University of South Wales, Pontypridd, CF37 1DL, UK \\ ${ }^{b}$ University of Southampton, University Rd, Southampton, SO17 1BJ, UK
}

\section{ARTICLE INFO}

\section{Keywords:}

Hydrogen cylinder

Heat transfer

Nusselt number

CFD

\begin{abstract}
A B S T R ACT
Fast-filling can lead to excessive heating of a hydrogen cylinder. Gas cylinders with large length-todiameter aspect ratios are prone to developing hot-spots that may cause the temperature of the structure locally to exceed specified limits. The hot-spots develop because convective heat transfer into the cylinder wall is not uniform along the length of the cylinder. Computational fluid dynamic simulations of fast-filling reveal that the flow within large aspect ratio cylinders is characterised by two distinct flow patterns. The region near to the inflow nozzle exhibits an axi-symmetric recirculating flow pattern that extends to approximately three cylinder diameters downstream from the inlet. Wall heat transfer in the recirculation region of the flow is driven strongly by the jet of turbulent fluid from the nozzle. The wall heat transfer in the recirculation region rapidly develops quasi-steady behaviour in which the Nusselt number is approximately proportional to the inlet jet Reynolds number. Downstream of the recirculation zone there is a region of axial flow, in which wall heat transfer occurs at a slower rate, driven by decaying turbulence transported from the recirculation zone. The reduced heat transfer in the end gas leads to a higher gas temperature but also to a lower structural temperature downstream of the recirculation zone. Analysis shows that wall heat transfer can be enhanced and peak gas temperatures reduced by dividing the hydrogen injection between separate nozzles. Directing the nozzles axially, ideally at intervals of around 4 cylinder diameters, increases heat transfer and prevents the formation of hot regions of stagnant fluid. This approach allows more hydrogen to be stored for a given pressure or gas temperature limit. However the analysis across various length-to-diameter ratios and nozzle configurations reveals substantially different trends for the gas temperatures appearing in ISO safety specifications, and the temperature of the structural materials they seek to safeguard.
\end{abstract}

\section{Introduction}

Global carbon dioxide emissions due to fossil fuels has significantly increased during the past century. According to Environmental Protection Agency, 95\% of the world's transportation energy is obtained from petroleum-based fuel and contributes to $14 \%$ of the global greenhouse gas emissions [1]. Thus, there is a need for the use of alternative fuels in the transportation sector in order to reduce the discharge of carbon dioxide into the atmosphere. Hydrogen can play a vital role as a clean energy carrier for vehicles since its oxidation only yields water vapour [2]. Gaseous storage is preferred currently for hydrogen powered vehicles, as opposed to either liquid hydrogen, which requires a cryogenic cooling system, or solid-state approaches, for example involving metal hydrides, whose implementation still requires further research [3]. In comparison to other gases, such as compressed natural gas, hydrogen has a much lower energy density for the same pressures and temperatures. Thus, hydrogen vessels on-board of vehicles are designed to contain the compressed hydrogen gas at pressures of $35 \mathrm{MPa}$ or 70 $\mathrm{MPa}$ [4]. Those relatively high pressures are required to re-

${ }^{\star}$ This document is the results of the research project funded by both the Engineering and Physical Sciences Research Council (EPSRC) and Luxfer Gas Cylinders Ltd.

* Corresponding author

vishagen. ramasamy@southwales.ac.uk (V. Ramasamy)

Onttp://staff. southwales.ac.uk/users/10711-vramasam (V.

Ramasamy)

ORCID(s): $0000-0003-3484-9646$ (V. Ramasamy) duce the volume of the storage vessels on-board, while storing a mass of hydrogen gas such that the range of the vehicle is on par to the conventional petrol vehicles [5].

Two types of cylinders are currently used as on-board storage for hydrogen-powered cars: Type III and Type IV. The structure of both cylinders consists of an outer laminate, which is made of a carbon fibre reinforced polymer (CFRP) to provide the structural strength and an inner liner whose main purpose is to prevent leakage [6]. The inner liner of the Type III cylinder consists of an aluminium alloy while plastic is used in the Type IV cylinder. There are currently two types of infrastructure that are used for the filling of hydrogen vehicles: time-fill and fast-fill [7]. Fleets such as buses with regular duty cycles typically use time fill stations in which compressors are used directly, for example, providing overnight filling of vehicles. Fast-fill implies that the cylinders in the vehicles are filled within three to five minutes. The rapid compression of the gas inside the cylinder combined with the negative Joule-Thompson coefficient of hydrogen at the filling pressures and temperatures leads to an increase in the gas temperature [8]. Fast-filling implies less time for this heat to be dissipated, resulting in an increase in temperature of the pressure vessel that could exceed the safe limits of its structural materials. As such, the maximum allowed temperature inside the vessel is set at $358 \mathrm{~K}$ by the International Standard Organisation, while the maximum allowable pressure is 1.25 times the cylinder's designed pressure [9].

Several CFD models for the fast-filling of hydrogen cylin- 
ders in either two dimensions (2D) or three dimensions (3D) $[8,10,11,12,13,14,15,16]$ have shown that the $k$ - $\varepsilon$ turbulence model [17] coupled with a real gas equation of state is capable of accurately predicting the average gas temperature during the fill. The CFD simulations were performed on cylinders that had the same geometry as the respective experiments and had relatively short aspect ratios with the inner length-to-diameter ratios of all cylinders being less than 2.5 . The simulations $[8,10,11,12,13,14,15]$ showed that the gas temperature within the cylinder is approximately homogeneous in all cases throughout the respective fills. Upon the validation of their CFD model, Zheng et al. [15], also performed a CFD simulation for the fast-filling of a cylinder with the inner length-to-diameter ratio set to 4.5. The simulation showed that the gas temperature within the high aspect ratio cylinder is no longer homogeneous and increases axially with the highest temperature occurring in the caudal region of the cylinder. However, Zheng et al. [15], in addition to changing the inner length-to-diameter ratios of the original cylinder from 2.3 to 4.5 , also increased the volume of the cylinder from $74 \mathrm{~L}$ to $150 \mathrm{~L}$. Similarly, Bourgeois et al. [18] performed CFD simulations on hydrogen cylinders with length-to-diameter ratios of 2.4, 2.7 and 5.4, while also changing the volume. CFD studies were also performed by $\mathrm{Li}$ et al. [16] on the fast-filling of hydrogen cylinders with varying aspect ratios and volumes. However, for a given mass flow rate, the density of the gas is smaller in larger cylinders, which results in the gas being less compressed and leads to lower temperatures during the filling. Thus, an understanding of the effect of aspect ratio of the cylinder was not properly established since two key geometric parameters of the original cylinders were altered in both cases.

The objective of the present study is to determine the effect of cylinder aspect ratio on the gas temperature and the flow field in the cylinder. Initially, a 2D axisymmetric simulation of the fast refuelling to a final pressure of $35 \mathrm{MPa}$ of $74 \mathrm{~L}$ hydrogen cylinder with the inner length-to-diameter ratio (L/D) of 2.4 is performed using the commercial software FLUENT based on the inlet conditions from the experiment of Dicken and Merida [10]. Once validated, simulations of the fast refuelling are performed on cylinders with same volume of $74 \mathrm{~L}$ but with the L/D increasing up to a value of 8. The flow field and the temperatures within the cylinders during the fill are analysed, following which, different filling strategies are discussed.

\section{Formulation and Methodology}

\subsection{Governing Equations}

The governing equations that are used in the two dimensional axisymmetric simulations in FLUENT are the unsteady Reynolds-Averaged Navier-Stokes compressible flow equations [19] and are shown in Eqs. 1-3,

$$
\frac{\partial \bar{\rho}}{\partial t}+\frac{\partial(\bar{\rho} \tilde{u})}{\partial x_{i}}=0
$$

$$
\begin{gathered}
\frac{\partial\left(\bar{\rho} \tilde{u}_{i}\right)}{\partial t}+\frac{\partial\left(\bar{\rho} \tilde{u}_{i} \tilde{u}_{j}\right)}{\partial x_{j}}=-\frac{\partial \bar{p}}{\partial x_{i}}+\frac{\partial}{\partial x_{j}}\left(\bar{\tau}_{i j}-\overline{\rho u_{i}^{\prime} u^{\prime}{ }_{j}}\right) \\
\frac{\partial\left(\bar{\rho} \tilde{H}_{i}\right)}{\partial t}+\frac{\partial\left(\bar{\rho} \tilde{H} \tilde{u}_{j}\right)}{\partial x_{j}}=-\frac{\partial \bar{p}}{\partial t}+ \\
\frac{\partial}{\partial x_{j}}\left(\bar{q}_{j}-\overline{\rho h^{\prime} u^{\prime}}{ }_{j}+\tilde{u}_{i} \bar{\tau}_{i j}+\overline{u_{i}^{\prime} \tau_{i j}}\right)
\end{gathered}
$$

where $\bar{\rho}$ is the ensemble-averaged density and $\tilde{u}$ is the Favreaveraged velocity. $\bar{\tau}_{i j}$ is the stress tensor. The unclosed terms $\overline{\rho u_{i}^{\prime} u_{j}^{\prime}}, \overline{\rho h^{\prime} u_{j}^{\prime}}$ and $\overline{u_{i}^{\prime} \tau_{i j}}$ are modelled using the $k$ - $\varepsilon$ turbulence model [17].

\subsection{Turbulence Model}

The closure strategy of the standard $k$ - $\varepsilon$ turbulence model is used to determine the unclosed terms in the fast filling simulations of the cylinders. The $k-\varepsilon$ model involves the PDE of the transport equations of the turbulent kinetic energy per unit mass $(k)$ and the dissipation rate per unit mass $(\varepsilon)$. The two-transport equations implemented in FLUENT are as follows [19]:

$$
\begin{gathered}
\frac{\partial\left(\rho k_{t}\right)}{\partial t}+\frac{\partial\left(\rho k_{t} u_{i}\right)}{\partial x_{i}}=\frac{\partial}{\partial x_{j}}\left[\left(\mu+\frac{\mu_{t}}{\sigma_{k}}\right) \frac{\partial k_{t}}{\partial x_{j}}\right] \\
+\mu_{t} S^{2}-\rho \epsilon-2 \rho \epsilon \frac{k_{t}}{c_{s}^{2}} \\
\frac{\partial\left(\rho \epsilon_{t}\right)}{\partial t}+\frac{\partial\left(\rho \epsilon_{t} u_{i}\right)}{\partial x_{i}}=\frac{\partial}{\partial x_{j}}\left[\left(\mu+\frac{\mu_{t}}{\sigma_{\epsilon}}\right) \frac{\partial \epsilon_{t}}{\partial x_{j}}\right] \\
+C_{1 \epsilon} \frac{\epsilon_{t}}{k_{t}} \mu_{t} S^{2}-C_{2 \epsilon} \rho \frac{\epsilon_{t}^{2}}{k_{t}}
\end{gathered}
$$

where $\mu$ is the dynamic viscosity, $\sigma_{k}$ and $\sigma_{\epsilon}$ are the turbulent Prandtl numbers for $k$ and $\epsilon$ respectively. In the simulations, the values of $\sigma_{k}$ and $\sigma_{\epsilon}$ were respectively set to 1 and 0.85 . $C_{1 \epsilon}$ and $C_{2 \epsilon}$ are constants with given values of 1.44 and 1.92 respectively [19]. $c_{S}$ is the speed of sound, $S$ is the modulus of the mean rate-of-strain tensor and $\mu_{t}$ is the eddy viscosity (Eq. 6).

$$
\mu_{t}=\bar{\rho} C_{\mu} \frac{k^{2}}{\epsilon}
$$

where $C_{\mu}$ is the default constant of the $k$ - $\varepsilon$ turbulence model and has a value of 0.09 .

\subsection{Equation of State}

The governing and turbulence model equations that have been previously described have to be closed using an equation of state. Since real gas effects are significant at the pressures and temperatures involved in the cylinders, the hydrogen gas is expected to deviate from ideal gas behaviour. The real gas equation chosen for the simulations is the Helmholtz 
Thermal response of high-aspect-ratio hydrogen cylinders undergoing fast-filling

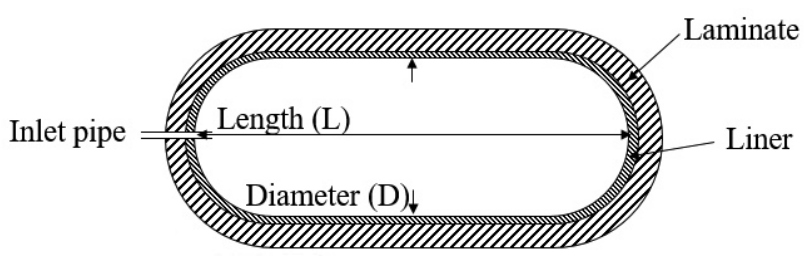

Figure 1: Schematic diagram of the hydrogen cylinder construction (not to scale).

equation of state for normal hydrogen of Leachman et al. [20], which is obtained from the NIST database: REFPROP 10.0 [21]. The Helmholtz equation of state [20] is applicable with a temperature range between $13.9 \mathrm{~K}$ and $1000 \mathrm{~K}$ along with maximum pressure of $1000 \mathrm{MPa}$.

\subsection{Molecular viscosity and thermal conductivity}

The molecular viscosity and thermal conductivity of the hydrogen gas are not constant throughout the filling process due to the effects of compressibility. Those respective values also obtained from REFPROP 10.0 [21]. The software REFPROP 10.0 [21] respectively uses the correlations of Muzny et al.[22] and Assael et al. [23] to determine the viscosity and thermal conductivity of the gas throughout the filling process.

\subsection{Cylinder Geometry and Computational Grid}

The 2D axisymmetric geometry of the cylinder is split into two domains: the fluid domain that consists of the hydrogen gas and the solid domain that involves the liner and the laminate illustrated in Fig. 1. The dimensions and material properties of the cylinder are displayed in Table 1 and Table 2 respectively. The simulations employ a structured body-fitted mesh. The mesh is refined in the injection pipe, the jet region and at the cylinder wall to resolve the viscous sublayer such that the maximum non-dimensional $\mathrm{y}^{+}$value is less than one.

Table 1

Dimension of the cylinder [10].

\begin{tabular}{lc}
\hline Description & Dimension $(\mathrm{m})$ \\
\hline Length of tank & 0.893 \\
Inner radius of tank & 0.179 \\
Outer radius of tank & 0.198 \\
Liner thickness & 0.004 \\
Laminate thickness & 0.015 \\
Inner diameter of inlet tube & 0.005 \\
Thickness of tube wall & 0.002 \\
Length of tube protruding into tank & 0.082 \\
\hline
\end{tabular}

\subsection{Initial and boundary conditions}

The initial pressure and temperature of the hydrogen gas are assumed to be uniform throughout the tank and are set to $9.36 \mathrm{MPa}$ and $293.4 \mathrm{~K}$ respectively, matching the experimental values of Dicken and Merida [10]. In addition, it is assumed that the liner and laminate are initially in thermal
Table 2

Material properties of the cylinder [10].

\begin{tabular}{llc}
\hline & Liner & Laminate \\
\hline Density $\left(\mathrm{kg} / \mathrm{m}^{3}\right)$ & 2730 & 1494 \\
Specific heat $(\mathrm{J} / \mathrm{kg} . \mathrm{K})$ & 900 & 938 \\
Thermal conductivity $(\mathrm{W} / \mathrm{m} . \mathrm{K})$ & 167 & 1.0 \\
\hline
\end{tabular}

equilibrium with the gas. The no slip boundary condition (v $=0 \mathrm{~m} / \mathrm{s}$ ) is used at the inner wall of the liner and the walls of the delivery pipe that are adjacent to the fluid. To enable heat transfer from the gas to the structure of the cylinder, the energy equation of the fluid is coupled to that of the inner wall of the liner. In addition, in order to determine the effect of heat transfer on the gas temperature, an adiabatic case is investigated whereby no conjugate heat transfer occurs from the gas to the walls of the cylinder. In the current model, heat transfer is assumed to be negligible between the fluid and the delivery pipe and thus, adiabatic wall conditions are applied to the walls of the pipe that are in direct contact with the fluid. Since the cylinder has an axisymmetric geometric, the axis boundary condition is used for its centreline. Pressure inlet boundary conditions are applied at the inlet of the cylinder. The total temperature and pressure at the inlet are both varied with time to match the experimental data of Dicken and Merida [10]. At the outer wall of the laminate and the delivery pipe two different cases are considered: isothermal conditions at a temperature of 293.4 $\mathrm{K}$ and adiabatic conditions. These two outer wall boundary conditions are chosen since they represent the two extreme scenarios whereby either maximum heat transfer occurs to the atmosphere (isothermal boundary condition) or no heat is transferred to the surroundings of the cylinder (adiabatic boundary condition).

\subsection{Solution convergence and grid independence}

The pressure-based solver with an implicit scheme is chosen for the simulation. The convergence of the mass flow rate at each time is monitored ensuing that the residual levels of the continuity equation reaches $10^{-4}$ which is similar to the residual convergence criteria that is suggested by the FLUENT guideline [19]. A time step of $10^{-4} \mathrm{~s}$ is chosen after confirming that further reduction of the time step to $10^{-5} \mathrm{~s}$ does not change the solution.

Grid independence is assessed considering the instantaneous velocity magnitude of the gas at two different vertical plane locations at an axial distance of $0.25 \mathrm{~L}$ and $0.75 \mathrm{~L}$ within the cylinder at different times during the fill. The grid independence tests are carried out whereby the grid distances are proportionally changed within the domain. The initial domain consists of 32841 cells and tests are further carried out on four other domains with cell counts of 49851, 72389, 99667 and 136433. Following the mesh sensitivity analysis, 99667 cells are used for the discretization of the axisymmetric domain of the cylinder for further simulations, since further increasing the cell count to 136433 does not lead to any changes in the instantaneous velocity field. 


\subsection{Changing the $L / D$ of the cylinder}

The length-to-diameter ratio of the cylinder is changed such that its value ranges between 3 and 8, while keeping the volume constant. An increase in the L/D of the cylinder involves an increase in the length of the cylinder coupled with a decrease in its diameter. Since a mesh independence test was already carried out on the original cylinder of 2.4 , the grid distances were proportionally increased in the axial direction of the cylinder with increasing L/D. In the lateral direction, the number of grid points is left unaltered for all cylinders. The inlet diameter is also kept constant for all the cylinders so that the fill time remains similar for a given inlet pressure profile. An increase in the L/D of the original cylinder also leads to an increase in the inner surface area of the cylinder and lowers the ratio $\left(D_{c y l} / D_{\text {inlet }}\right)$ of the inner diameter of the cylinder to the diameter of the inlet tube (Table 3).

Table 3

Inner surface areas and the ratio $D_{c y l} / D_{\text {inlet }}$ of the cylinders having different $L / D$.

\begin{tabular}{ccc}
\hline L/D & Surface area $\left(\mathrm{m}^{2}\right)$ & $D_{c y l} / D_{\text {inlet }}$ \\
\hline 2.4 & 0.962 & 79.2 \\
3 & 1.015 & 73.2 \\
4 & 1.094 & 66.6 \\
5 & 1.165 & 62.1 \\
6 & 1.228 & 58.6 \\
7 & 1.286 & 56.0 \\
8 & 1.338 & 53.8 \\
\hline
\end{tabular}

\section{Results}

Figure 2 shows that the boundary condition at the outer wall of the laminate does not play a role in the heat transfer from the gas to the structure of the cylinder. The massaveraged gas temperature is similar for either outer wall boundary conditions due to the low thermal conductivity of the laminate. The mass-averaged gas temperature that is obtained by the CFD models for both outer wall boundary conditions accurately predicts the rise in gas temperature when compared to the experiment. Only a slight discrepancy in gas temperatures is observed during the first few seconds at the start of the fill. The 2D CFD simulations also shows substantial heat transfer to the structure of the cylinder during the fast fill since, in the purely adiabatic case, the gas temperature in the cylinder significantly exceeds the gas temperature that was obtained from the experiment. In addition, without heat transfer, the gas temperature in the cylinder exceeds the maximum allowable limit of $358 \mathrm{~K}$.

\subsection{Inlet Reynolds number v/s Nusselt number}

The inlet pipe Reynolds number and the Nusselt number describing convective heat transfer on the inside of the cylinder are defined in Eqs. 7 and 8 respectively.

$$
R e=\frac{\rho V_{\text {inlet }} D_{\text {inlet }}}{\mu}
$$

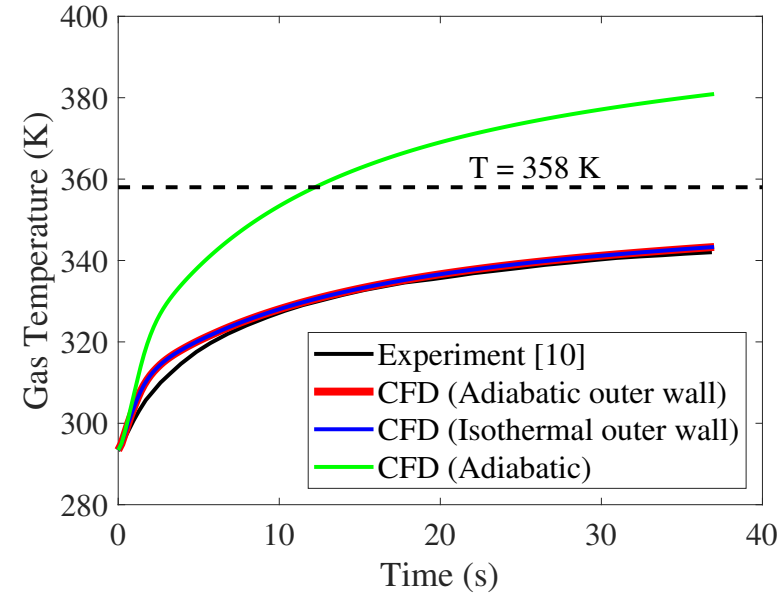

Figure 2: Comparison of the mass-average gas temperatures.

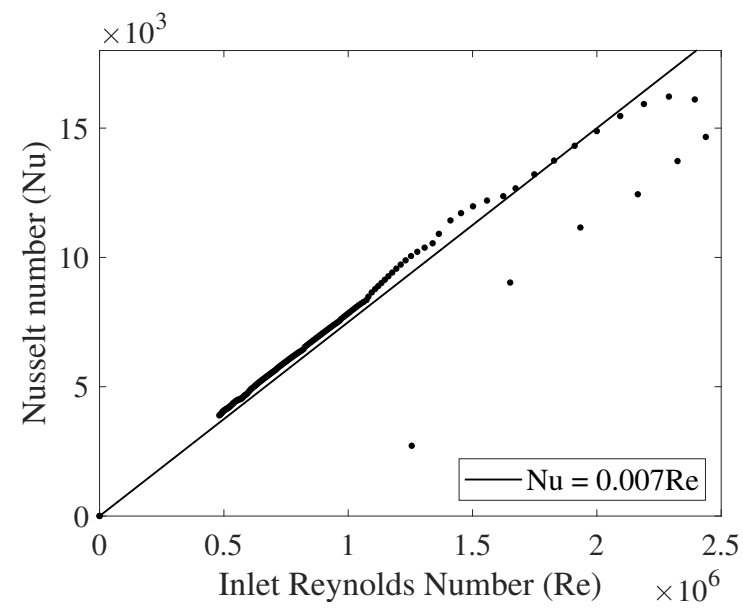

Figure 3: Nusselt number v/s Inlet Reynolds number.

where $\rho$ is the density of the gas at the inlet, $V_{\text {inlet }}$ is the bulk velocity at the inlet and $D_{\text {inlet }}$ is the inlet diameter and $\mu$ is the dynamic viscosity

$$
N u=\frac{h D_{c y l}}{\lambda}
$$

where $h$ is the heat transfer coefficient, $D_{c y l}$ is the diameter of the cylinder and $\lambda$ is the gas thermal conductivity. The heat transfer coefficient $(h)$ is obtained from Eq. 9.

$$
\dot{q}=h\left(T_{w}-T_{g}\right)
$$

where $\dot{q}$ is the area-averaged heat flux at the inner wall of the cylinder, $T_{w}$ is the area-averaged wall temperature and $T_{g}$ is the mass-averaged gas temperature.

The Nusselt number describing convective heat transfer on the inside of the cylinder is plotted versus the inlet pipe Reynolds number in Fig. 3, with points shown at $0.2 \mathrm{sec}-$ ond intervals. It is observed that after two seconds into the fill, there is a near-linear relationship between Nusselt number and Reynolds number. The two second period that it takes to establish a quasi-steady linear relationship between 
Thermal response of high-aspect-ratio hydrogen cylinders undergoing fast-filling
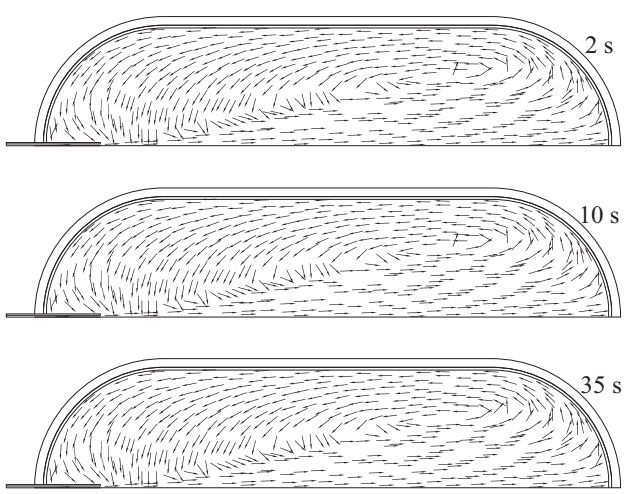

(a)

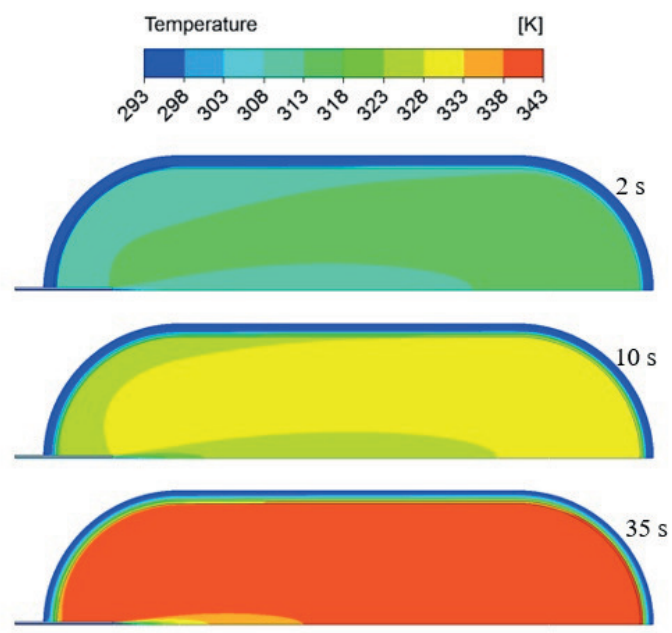

(b)

Figure 4: (a) Flow field of the gas in the cylinder, (b) Gas and structural temperature in the cylinder.

Nusselt and Reynolds numbers corresponds to the time taken to establish the large-scale recirculating flow pattern shown in Fig. 4(a). Figure 4(a) shows that the velocity field, normalised by the inlet velocity remains similar throughout the quasi-steady period starting after 2 seconds into the fill. The gas from the inlet hits the opposite end of the tank and turns along the surface of the inner wall towards the inlet. Largescale mixing due to the recirculating flow field results in a near-uniform gas temperature within the bulk of the cylinder during the fill (Fig. 4(b)).

\subsection{Effect of increasing the $L / D$ of the cylinder}

The mass-averaged gas temperature within the cylinder does not change significantly when the L/D of the cylinder varies between 2.4 and 5 (Fig. 5(a)) despite an increase in the surface area-to-volume ratio of the cylinder. A further increase in $\mathrm{L} / \mathrm{D}$ beyond a value of 5 increases the final massaveraged temperature by up to $10 \mathrm{~K}$ (Fig. 5(a)), with the local peak gas temperature increasing by up to $70 \mathrm{~K}$ (Fig. $5(b))$ at $\mathrm{L} / \mathrm{D}=7$. The increasingly large temperature variation within the cylinder means that individual localised temperature measurements are not sufficient to characterise the temperature field in large $\mathrm{L} / \mathrm{D}$ cylinders. The dependence of

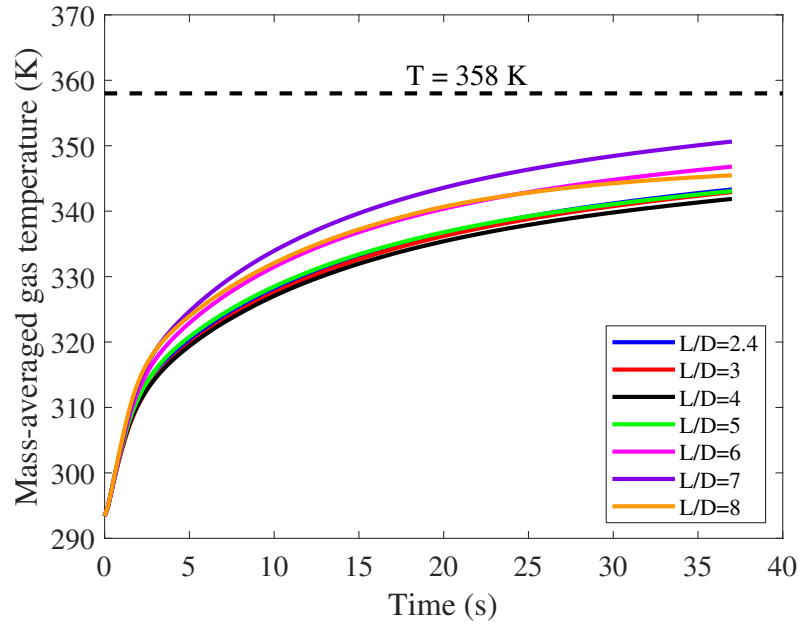

(a)

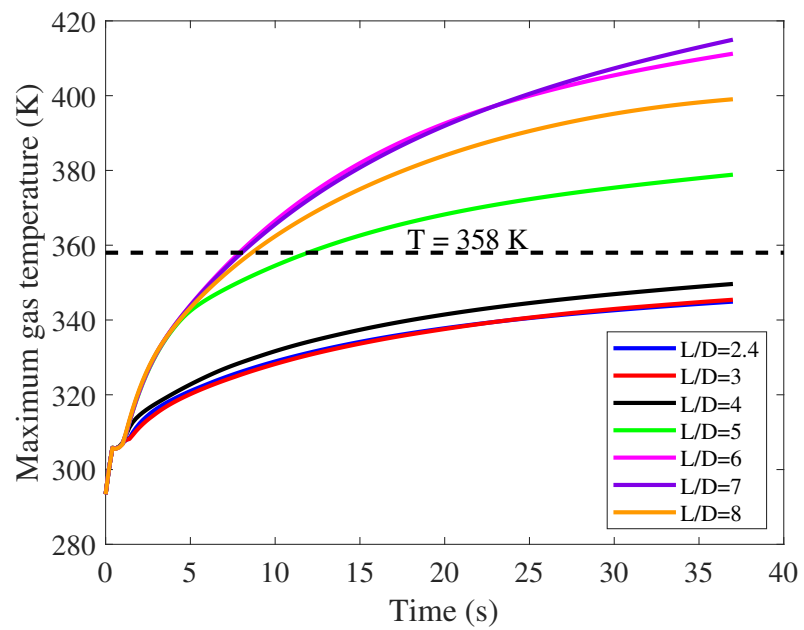

(b)

Figure 5: (a) Comparison of the mass-averaged gas temperature in cylinders with different L/Ds, (b) Comparison of the local maximum gas temperatures in cylinders with different L/Ds.

the mass-averaged and peak gas temperatures with L/D are shown at the end of the 37 second fill in Fig. 6. Beyond $\mathrm{L} / \mathrm{D}=7$, the incremental increase in surface area-to-volume ratio enhances heat transfer and counteracts the increase in gas temperature due to the compression of the gas in the caudal region of the cylinder. This leads to a reduction of the peak gas temperature as well as the inhomogeneity in gas temperature. Fig. 6 also shows the peak material temperature for cylinders with different $\mathrm{L} / \mathrm{D}$ at the end of the 37 second fill. Counter to the trend for gas temperature, the maximum structural temperature decreases with increasing $\mathrm{L} / \mathrm{D}$ as a result of the effect of $\mathrm{L} / \mathrm{D}$ on the flow field and the consequent reduction in the rate of heat transfer.

Fig. 7(a) shows the contours of gas temperature thirty seconds into the fill for cylinders with L/D from 2.4 to 8 . The temperature field is largely homogeneous for for cylinders with $\mathrm{L} / \mathrm{D}$ up to 3 . For larger $\mathrm{L} / \mathrm{D}$, a region of homoge- 
Thermal response of high-aspect-ratio hydrogen cylinders undergoing fast-filling

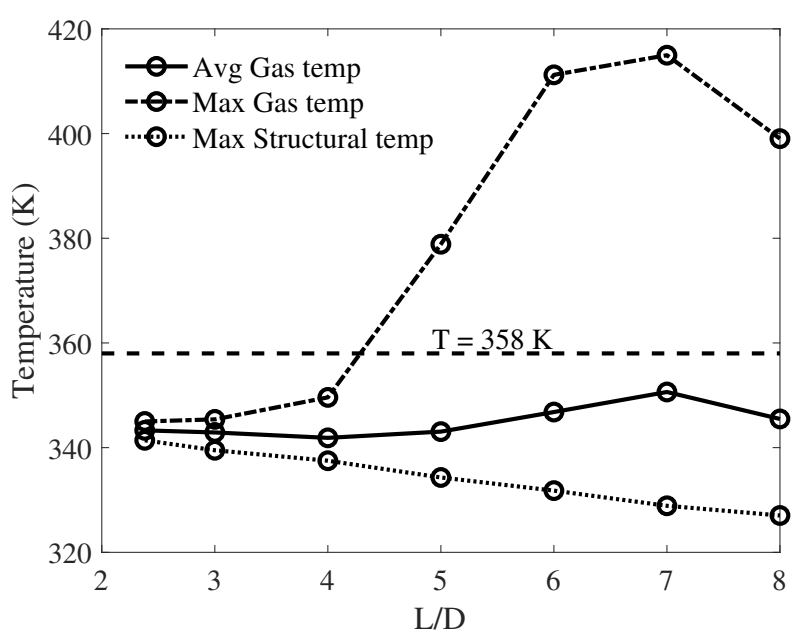

Figure 6: Mean and peak gas temperature and peak material temperature for cylinder with different $L / D$ at end of the 37 second fill.

neous temperature (Zone 1) remains in the first 3 diameters from the inlet but the difference between mass-averaged and peak temperatures increases due to development of highertemperatures in a second region (Zone 2) extending from 3 diameters downstream. This finding is similar to the CFD simulations performed by Bourgeois et al. [18].The corresponding plots of velocity vectors in Fig. 7(b) illustrate that the homogeneous temperature Zone 1 coincides with a region of recirculating flow. The recirculating flow pattern and high turbulence levels from the inlet jet enhance thermal mixing in Zone 1. The simulations showed that the axial distance of the recirculating zone from the inlet is independent the incoming jet provided that the ratio of the cylinder diameter to the inlet diameter $\left(D_{c y l} / D_{\text {inlet }}\right)$ is between 53.8 and 79.2. The centre of the recirculating zone corresponds to a local point whereby the gas velocity is zero. A straight line is drawn from exit of the delivery pipe to the particular local point at the centre of the recirculating zone in each cases and the resulting opening jet spread angle $(\alpha)$ is determined from the horizontal. The jet spread angle varies between between $12.1^{\circ}$ and $12.5^{\circ}$ for the cylinders with varying L/D (Table 4). The average jet spread angle of $12.3^{\circ}\left(+/-0.2^{\circ}\right)$ found in this study is consistent with the spread angle of turbulent axisymmetric submerged jets [24]. Thus, the nearfield behaviour of the jet is similar to behaviour of a free jet. This may be expected since the momentum of the jet is much greater than that associated with the recirculation. In Zone 2 the flow pattern is effectively axial, trapping hot fluid in the caudal region and providing low levels of turbulent mixing. Due to the low rates of heat transfer, the fluid in the caudal region continues to get hotter due to compression heating.

Fig. 8(b) shows the heat flux at the inner wall of the cylinder at different times into the fill for the cylinder having a L/D of 8 . The heat flux is significantly higher in Zone 1 compared to Zone 2 during the first 10 seconds of the fill. The higher and approximately uniform heat transfer coefficient in Zone 1 is caused by recirculation of highly turbulent
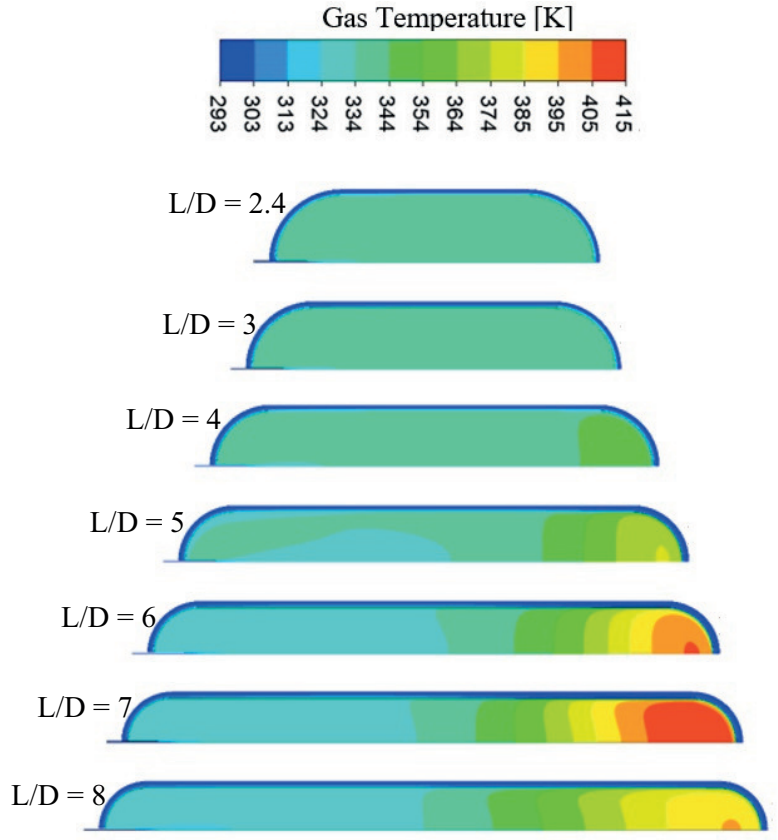

(a)

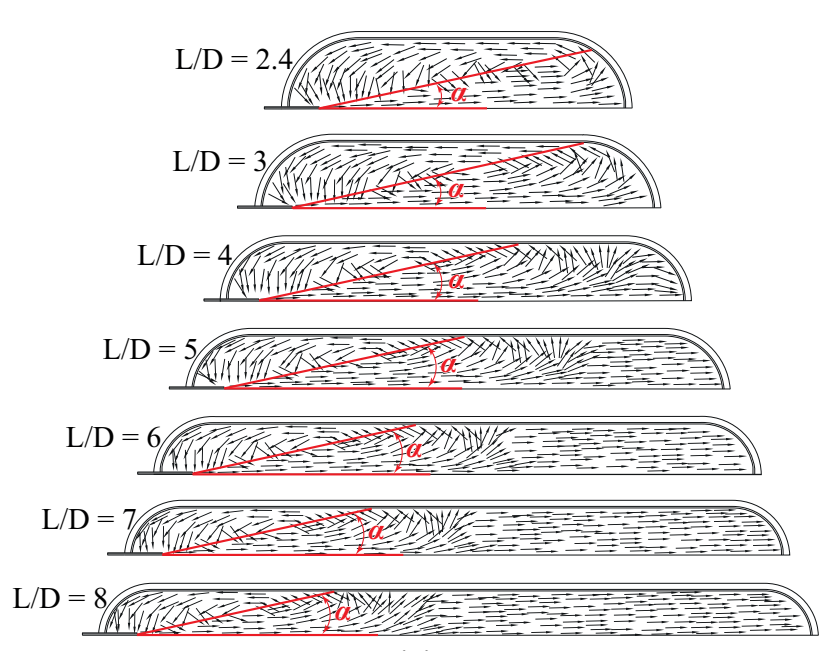

(b)

Figure 7: (a) Comparison of the contours of gas temperature $30 \mathrm{~s}$ into the fill for the different cylinders with varying L/D, (b) Flow field within the cylinders with varying $L / D$ at the fill time of $30 \mathrm{~s}$ with the corresponding jet angle $(\alpha)$.

jet fluid, which increases the heat transfer from the gas to the structure of the cylinder and results in a lower gas temperature in Zone 1. For fill times beyond 10 seconds, the heat flux is uniform irrespective of the flow field within the cylinder.

The cylinder-average Nusselt number is evaluated for the $\mathrm{L} / \mathrm{D}=5$ and 8 cylinders and plotted at $0.5 \mathrm{~s}$ intervals in Fig. 9(a). The cylinder-averaged Nusselt numbers exhibit a linear variation with jet inlet Reynolds number during the quasisteady portion of the fill for both $\mathrm{L} / \mathrm{D}=5$ and 8 , but with different gradients. Evaluating an effective Nusselt number 
Table 4

Jet angle $(\alpha)$ during the filling of the cylinders having different L/D.

\begin{tabular}{ll}
\hline L/D & Jet spread angle $(\alpha)$ \\
\hline 2.4 & $12.2^{\circ}$ \\
3 & $12.5^{\circ}$ \\
4 & $12.2^{\circ}$ \\
5 & $12.1^{\circ}$ \\
6 & $12.3^{\circ}$ \\
7 & $12.3^{\circ}$ \\
8 & $12.4^{\circ}$ \\
\hline
\end{tabular}

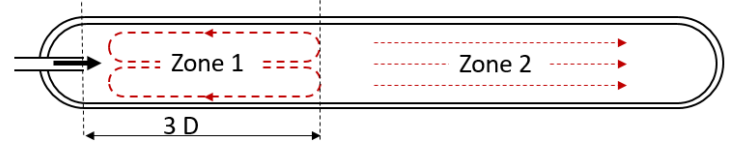

(a)

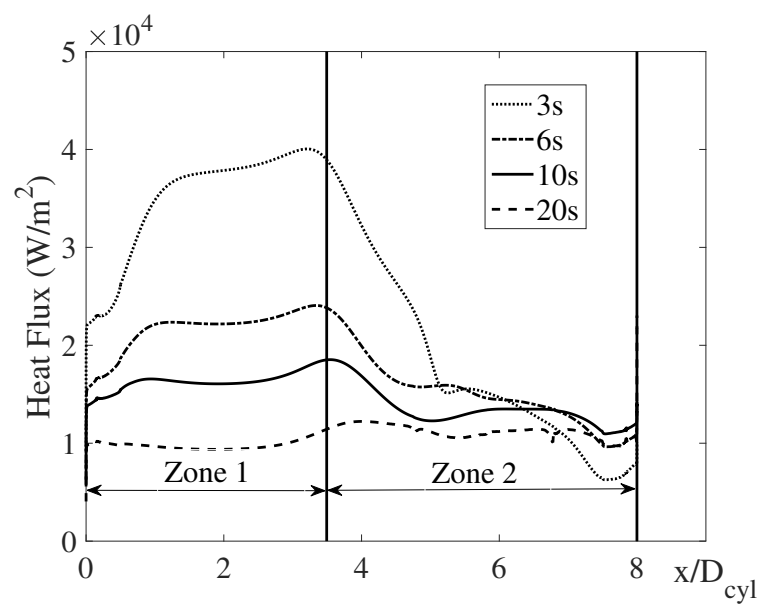

(b)

Figure 8: (a) Schematic of the 2 fluid regions within the cylinder with a $L / D>3$, (b) Heat flux at the inner wall of the cylinder with L/D of 8 at different times during the fill

based just on the heat transfer from Zone 1, it is found that the the Zone 1 Nusselt numbers (Fig. 9(b)) follow the same linear relationship with inlet Reynolds number for all L/D studied, including the $\mathrm{L} / \mathrm{D}=2.4$ case shown in Fig. 3. The Nusselt number in Zone 2 also follows a linear relationship with the inlet Reynolds number, but with a different constant of proportionality that depends on the length of that zone. While single-zone models have been applied successfully to model the thermal response of low L/D hydrogen cylinders [25], the difference in both Nusselt number and gas temperature between Zone 1 and Zone 2 motivates development of two-zone or multi-zone models to describe the thermal response of cylinders with $\mathrm{L} / \mathrm{D}>3$.

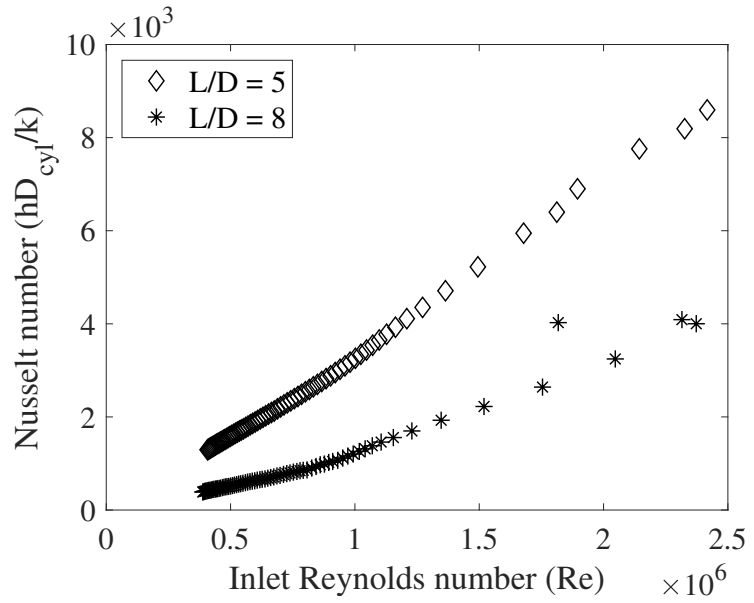

(a)

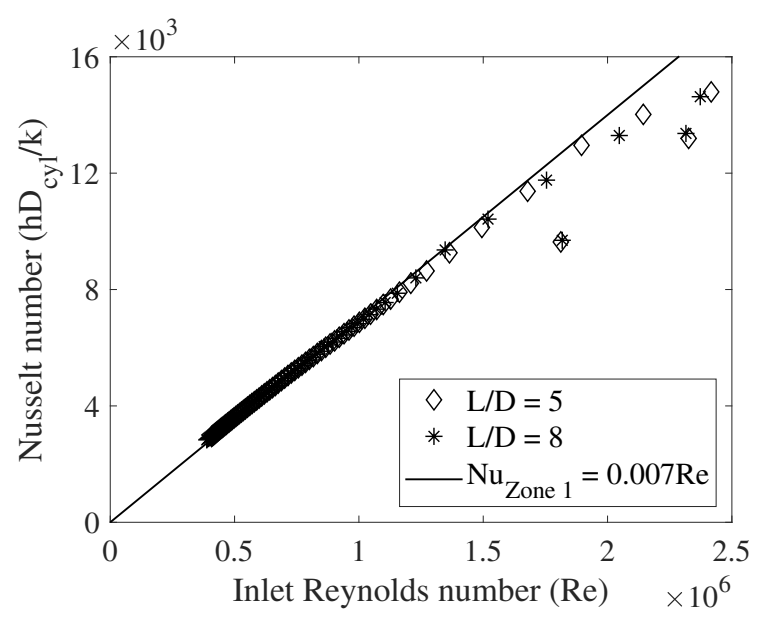

(b)

Figure 9: Nusselt number $v / s$ Inlet Reynolds number for cylinders with L/D of 5 and 8: (a) single-zone model, (b) Zone 1 only.

\subsection{Alternative nozzle configurations}

Alternative nozzle configurations are considered as a means of achieving more uniform gas temperatures and heat transfer to the cylinder walls. Since the preceding results indicate that axial fluid injection sets up a recirculation region of approximately 3 cylinder diameters in length, the approach taken is to inject fluid from multiple locations in order to avoid any large regions of stagnant fluid. In addition to the standard single-inlet configuration (Case 1), two additional configurations and their intended flow patterns are illustrated in Fig. 10: Case 2 introduces fluid as jets issuing inwards from both ends of the cylinder; Case 3 introduces fluid from two co-aligned jets, with one issuing near the left hand end of the cylinder and the other issuing from either $\mathrm{L}_{2}$ $=4 \mathrm{D}($ Case $3-\mathrm{A})$ or $\mathrm{L}_{2}=5 \mathrm{D}$ (Case 3-B) from the left hand end of the cylinder, where $L_{2}$ is the distance from the left hand end of the cylinder to the nozzle outlet. The fast-filling process is simulated for the additional cases, considering an overall cylinder aspect ratio of $\mathrm{L} / \mathrm{D}=8$ and total inlet cross sectional area of $19.6 \mathrm{~mm}^{2}$, which is equivalent to a single 
Thermal response of high-aspect-ratio hydrogen cylinders undergoing fast-filling

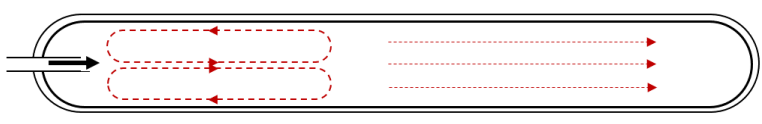

(a)

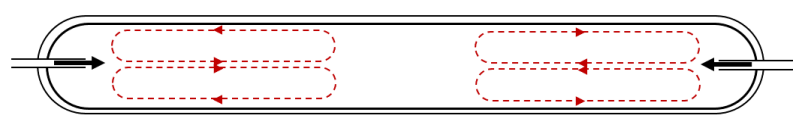

(b)

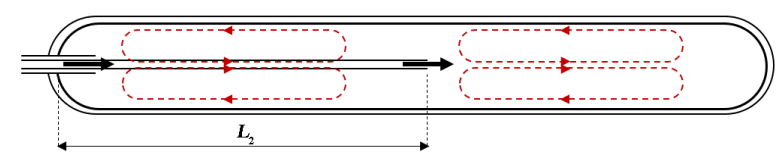

(c)

Figure 10: Three alternative injection nozzle configurations: (a) Case 1; (b) Case 2; (c) Case 3.

Table 5

Comparison of the final mass-averaged gas temperatures and final mass of gas in the cylinder $(L / D=8)$ using the different inlet configurations.

\begin{tabular}{lcccc}
\hline & Case 1 & Case 2 & Case 3-A & Case 3-B \\
\hline $\begin{array}{l}\text { Final average } \\
\text { gas temperature (K) }\end{array}$ & 344.4 & 338.6 & 340.1 & 341.1 \\
$\begin{array}{l}\text { Final mass } \\
\text { of gas (kg) }\end{array}$ & 1.534 & 1.556 & 1.549 & 1.545 \\
$\begin{array}{l}\% \text { increase in } \\
\text { final mass of gas }\end{array}$ & - & $1.4 \%$ & $1.0 \%$ & $0.7 \%$ \\
\hline
\end{tabular}

inlet pipe of $7.07 \mathrm{~mm}$ diameter. The cross-sectional area of the inner and outer tubes in Case 3-A and 3-B are $9.8 \mathrm{~mm}^{2}$ each. This ensures that the mass flow rates and the velocity is similar in both the inner and outer tubes of Cases 3-A and 3-B. Separate grid-resolution analysis is not considered necessary for Cases 2, 3-A and 3-B since the most severe resolution requirement is within the near-field region of the jet. Grid characteristics are identical within the near-field region of the jet for all three cases and the rest of the domain is expected to be over-resolved.

The mass-averaged and peak temperatures for Cases 1, 2, 3-A and 3-B during the fill are shown in Fig. 11. The multiple-inlet cases give more homogeneous temperature fields with peak temperature reduced by $30-40 \mathrm{~K}$ and mean temperature reduced by around $6 \mathrm{~K}$ compared to the single-inlet Case 1. The greatest temperature reduction is for the symmetrical Case 2. Table 5 reports the mass stored for each case at the end of the $37 \mathrm{~s}$ fill, showing that the temperature reduction in Case 2 gives a $1.4 \%$ increase in the mass of gas stored. The temperature field and velocity vector plots in Fig. 12 confirm that the two-nozzle configurations avoid stagnant high-temperature regions by establishing two distinct recirculation zones.

Configuration 2 requires modification of the cylinder struc-

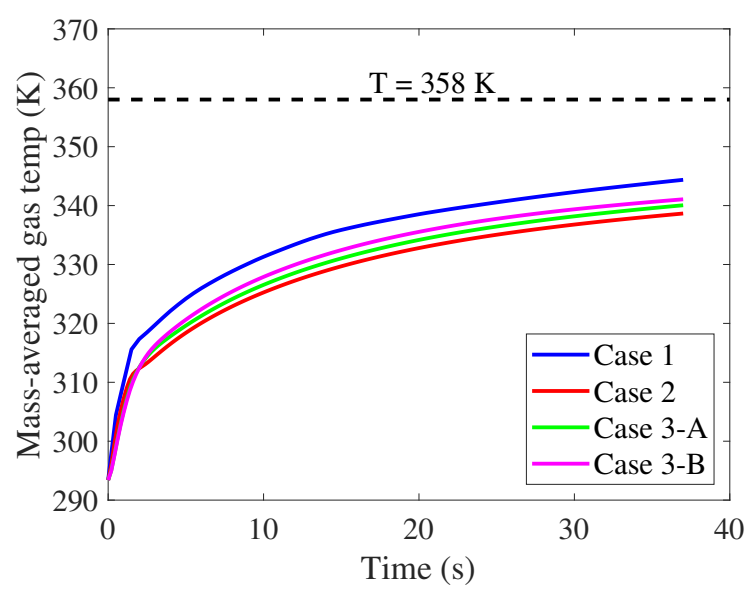

(a)

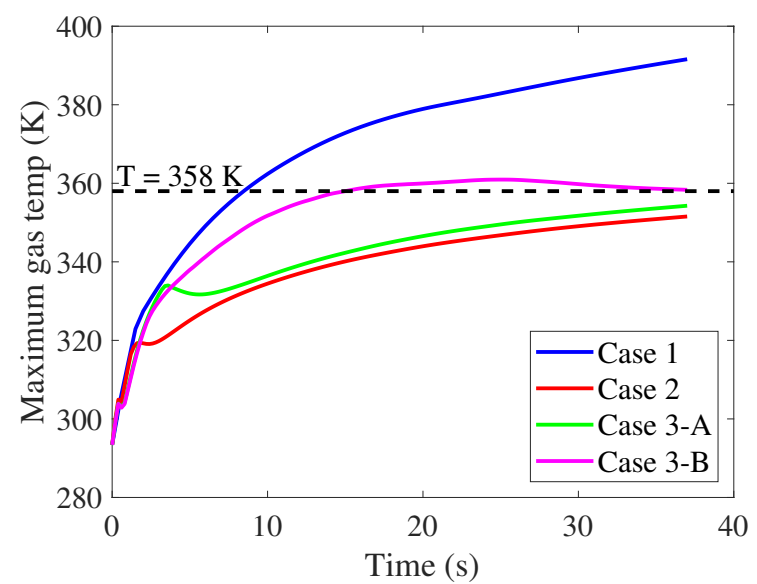

(b)

Figure 11: (a) Comparison of the mass-averaged gas temperatures in the cylinder $(L / D=8)$ with different inlet configurations, (b) Comparison of the maximum local gas temperatures in the cylinder $(L / D=8)$ with different inlet configurations.

tural design in order to incorporate two separate inflow nozzles, possibly increasing expense. Configuration 3-A achieves a similar thermal performance to Case 2 without the need for a second opening in the cylinder, since two inlet pipes may be introduced concentrically from a single end, potentially reducing cost. Comparison of the results for Cases 3-A and 3 -B show that increasing the spacing between the two inlets from 4D apart (Case 3-A) to 5D apart (Case 3-B) leaves a small hot-spot of fluid close to the centre of the cylinder, indicating that a nozzle separation of $4 \mathrm{D}$ is an upper limit for avoiding hot-spots in the fluid. The modifications of the nozzle configuration (Cases 2, 3-A \& 3-B) lead to a reduction of the hot-spots that is observed in Case 1. This is due to the enhanced heat transfer from the gas to the structure of the cylinder, which results in an increase in the maximum temperature of the material of the cylinder (Fig. 13) and can potentially diminish its structural integrity. 
Thermal response of high-aspect-ratio hydrogen cylinders undergoing fast-filling

Gas Temperature $\lceil\mathrm{K}\rceil$

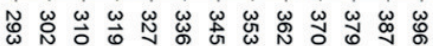

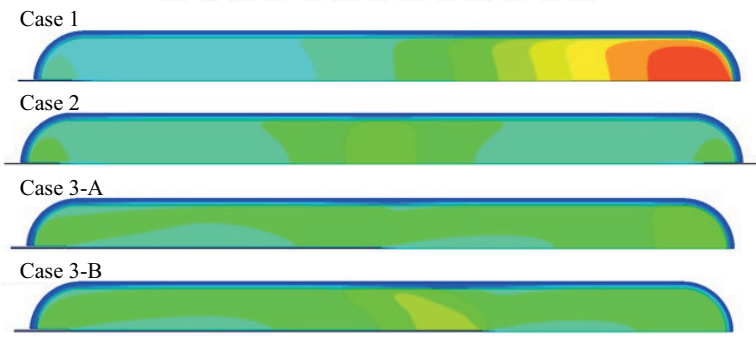

(a)

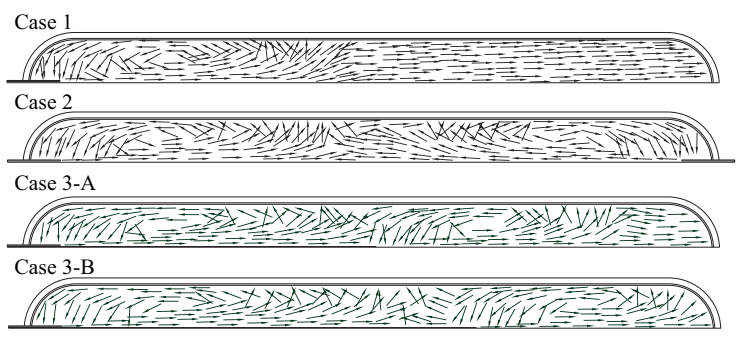

(b)

Figure 12: (a) Comparison of the contours of gas temperature thirty seconds into the fill in the cylinder $(L / D=8)$ with the different inlet configurations, (b) Flow field within the cylinders $(L / D=8)$ with the different inlet configurations at the fill time of thirty seconds.

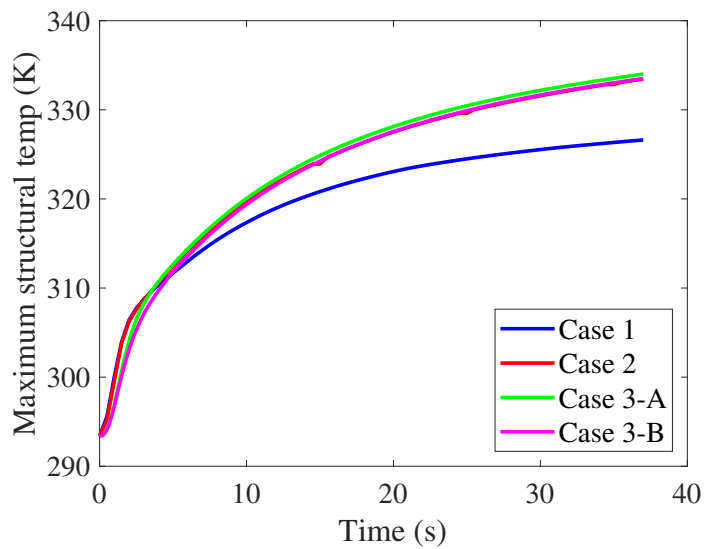

Figure 13: Comparison of the maximum temperature of the structure of the cylinder $(L / D=8)$ throughout the with the different inlet configurations.

\section{Conclusion}

The thermal response of high-aspect ratio hydrogen storage cylinders during fast filling has been investigated using numerical simulations. It is found that two-dimensional axisymmetric Reynolds-Averaged Navier Stokes simulations accurately reproduce experimental results for a 74 litre test case. Simulations across a range of L/D aspect ratios lead to the following conclusions:

- Axial fluid injection leads to a recirculating region of flow (Zone 1) extending to three cylinder diameters from the inlet.

- For cylinders more than three diameters in length, a region of axial flow (Zone 2) exists downstream of Zone 1.

- Heat transfer in Zone 1 is driven by recirculation of turbulence from the incoming jet of hydrogen for the first 10 seconds of the fill. The heat flux along the length of the cylinder is uniform after the initial 10 seconds.

- The Zone 1 Nusselt number is approximately proportional to the inlet Reynolds number and independent of $\mathrm{L} / \mathrm{D}$.

- Heat transfer in Zone 2 is slower, resulting in higher local gas temperature but also locally lower wall temperatures.

- Single-zone thermal modelling assumptions should not be applied to computational or experimental characterisation of cylinders with L/D greater than 3 .

- In cylinders with $\mathrm{L} / \mathrm{D}$ greater than 3 , local or average gas temperature values used in, for example, International Standard Organisation regulations for the filling of light duty hydrogen vehicles, do not follow a direct relationship with the temperature of the structural materials those regulations seek to safeguard.

Two alternative nozzle configurations are investigated as a means to achieve more homogeneous temperature and heat transfer. For a cylinder with $\mathrm{L} / \mathrm{D}=8$, it is possible to avoid regions of stagnant or axial flow by injecting fluid through two nozzles rather than one:

- Injecting fluid equally through opposite ends of the cylinder resulted in a $35 \mathrm{~K}$ reduction in the peak gas temperature and a $1.4 \%$ increase in the stored mass of hydrogen at the end of the fast fill compared to a single-inlet fill.

- A similar improvement can also be achieved by introducing two, possibly concentric, inlet pipes through one end of the cylinder, thereby avoiding the need to modify the cylinder design to introduce a second opening.

- Given that the natural length of the recirculation zones is three diameters, the nozzles should be spaced to achieve recirculation zones no more than four diameters apart in order to avoid hot regions of stagnant flow.

\section{References}

[1] EPA, Greenhouse gas emissions, https://www.epa.gov/ ghgemissions/global-greenhouse-gas-emissions-data (2017). 
[2] L. Wang, C. Zheng, B. Wang, Z. Wei, Thermo-mechanical investigation of composite high-pressure hydrogen storage cylinder during fast filling, Int J Hydrogen Energ. 40 (2015) 6853-6859.

[3] P. L. Woodfield, M. Masanori, Y. Mitsutake, Measurement of averaged heat transfer coefficients in high-pressure vessel during charging with hydrogen, J Sci and Tech. 2 (2007) 180191.

[4] SAE, Fueling protocols for light duty gaseous hydrogen surface vehicles, http://standards.sae.org/j2601_201003/ (2010).

[5] Toyota, Powering the future - hydrogen fuel cell vehicles could change mobility forever, http://www.toyota-global. com/innovation/environmental_technology/fuelcell_vehicle/ (2016).

[6] S. C. Kim, S. H. Lee, K. B. Yoon, Thermal characteristics during hydrogen fueling process of type iv cylinder, Int J Hydrogen Energ. 35 (2010) 6830-6835.

[7] W. Liss, M. Czachorski, K. Kountz, Sizing and operating considerations for natural gas and hydrogen fueling systems, https://www.gti .energy (2015).

[8] A. Suryan, H. Kim, T. Setoguchi, Three dimensional numerical computations on the fast filling of a hydrogen tank under different conditions, Int J Hydrogen Energ. 37 (2012) 7600 7611.

[9] ISO, Gaseous hydrogen and hydrogen blends - land vehicle fuel tanks, iso/ts 15869, https://www. iso.org/standard/ 52871. html (2009).

[10] C. J. B. Dicken, W. Merida, Modelling the transient temperature distribution within a hydrogen cylinder during refueling, Numerical Heat Transfer, Part A: J Comp and Meth. 53:7 (2007) 685-708.

[11] A. Suryan, H. D. Kim, T. Setoguchi, Comparative study of turbulence models performance for refueling of compressed hydrogen tanks, Int J Hydrogen Energ. 38 (2013) 9562-9569.

[12] J. Zheng, J. Guo, J. Yang, Y. Zhao, X. Pan, L. Zhang, L. Zhao, Investigations on temperature variation within a type iii cylinder during the hydrogen cycling test, Int J Hydrogen Energ. 39 (2014) 13926-13934.

[13] D. Baraldi, D. Melideo, M. Galassi, R. Cebolla, B. Iborra, P. Moretto, Cfd model performance benchmark of fast filling simulations of hydrogen tanks with pre-cooling, Int J Hydrogen Energ. 39 (2014) 4389-4395.

[14] T. Johnson, T. Bozinoski, J. Ye, O. Sartor, J. Zheng, J. Yang, Thermal development and validation for rapid filling of high pressure hydrogen tanks, Int J Hydrogen Energ. 40 (2015) 9803-9814.

[15] J. Zheng, J. Guo, J. Yang, Y. Zhao, X. Pan, L. Zhang, L. Zhao, Experimental and numerical study on temperature rise within a 70 mpa type iii cylinder during fast refueling, Int J Hydrogen Energ. 38 (2013) 10956-10962.

[16] Q. Li, J. Zhou, Q. Chang, W. Xing, Effects of geometry and inconsistant mass flow rate on temperatures within a pressurized hydrogen cylinder during refueling, Int J Hydrogen Energ. 37 (2012) $6043-6052$.

[17] B. Launder, D. Spalding, Lectures in mathematical models of turbulence., London; New York : Academic Press, 1972.

[18] T. Bourgeois, T. Brachmann, F. Barth, F. Ammouri, D. Baraldi, B. Melideo, D. Acosta-Iborra, D. Zaepffel, D. Saury, D. Lemonnier, Optimization of hydrogen vehicle refuellingrequirements, Int J Hydrogen Energ. 42 (2017) 13789 -13790 .

[19] ANSYS., Ansys fluent theory guide. (2017).

[20] J. W. Leachman, R. T. Jacobsen, S. G. Penoncello, E. W. Lemmon, Fundamental Equations of State for Parahydrogen, Normal Hydrogen, and Orthohydrogen, Journal of Physical and Chemical Reference Data 38 (3) (2009) 721-748.

[21] E. Lemmon, I. Bell, M. Huber, M. McLinden, Nist standard reference database 23: Reference fluid thermodynamic and transport properties-refprop, version 10.0, national institute of standards and technology, standard reference data program. (2018).

[22] C. Muzny, M. Huber, A. Karakov, Correlation for the viscosity of normal hydrogen obtained from symbolic regression., Journal of Chemical and Engineering Data. 58.4 (2013) 969979.

[23] M. Assael, J. Assael, M. Huber, R. Perkins, Y. Takata, Correlation of the thermal conductivity of normal and parahydrogen from the triple point to $1000 \mathrm{k}$ and up to $100 \mathrm{mpa}$, J. Phys and Chem. Ref.Data(JPCRD). 40 (2011) 033101-1 - 033101-13.

[24] G. Abramovich, The theory of turbulent jets, M.I.T. Press, Cambridge, Massachusetts, 1963, Ch. 12, p. 509.

[25] V. Ramasamy, E. S. Richardson, P. Reed, W. Hepples, A. Wheeler, Use of phase-change materials for temperature control during fast filling of hydrogen cylinders, High Temperature Material Processes: An International Quarterly of High-Technology Plasma Processes 22 (2) (2018). 